\title{
A New Macro Model Considering the Average Speed of Preceding Vehicles Group in CPS Environment
}

\author{
Yi-rong Kang, ${ }^{1,2}$ Di-hua Sun,,2 and Shu-hong Yang ${ }^{3}$ \\ ${ }^{1}$ Key Laboratory of Dependable Service Computing in Cyber Physical Society (Chongqing University), \\ Ministry of Education, Chongqing 400030, China \\ ${ }^{2}$ College of Automation, Chongqing University, Chongqing 400030, China \\ ${ }^{3}$ School of Computer Science, Guangxi University of Science and Technology, Liuzhou, Guangxi 545006, China
}

Correspondence should be addressed to Di-hua Sun; 719133604@qq.com

Received 14 December 2014; Accepted 6 February 2015

Academic Editor: Maria Gandarias

Copyright (C) 2015 Yi-rong Kang et al. This is an open access article distributed under the Creative Commons Attribution License, which permits unrestricted use, distribution, and reproduction in any medium, provided the original work is properly cited.

A new car following model considering the effect of average speed information of preceding vehicles group in real traffic is presented. Based on the new car following model, a new macro model for traffic flow is proposed employing the relationship between the micro and macro variables. The linear stability condition of the macro model is obtained by using the linear stability theory. The numerical tests show that the new model can not only simulate the dynamic process of shock, rarefaction wave, and small perturbation, but also can further stabilize the traffic flow.

\section{Introduction}

Traffic jams has attracted much attention of physicists and engineers. Various traffic models [1-6], including microscopic models and macroscopic models from different scales, have been proposed to describe the complex traffic phenomena. The physical mechanisms of traffic phenomena such as the stop-and-go traffic, the ghost jams, local clusters, and others are revealed. However, these models are unsuited to describe the complex phenomena resulting from the effects of average speed of preceding vehicles group (called local average speed, LAS), since they do not involve the factors. In reality, as we know, along with the remarkable development of the wireless communication technology, the transportation cyber physical systems (T-CPS) are now becoming widely available. T-CPS enable exchanging the velocity information among vehicles and provide average speed about preceding cars on road. Depending on this on-line traffic data, drivers can adjust the speed to the optimal state in advance.

In fact, LAS is described as the key parameter to reflect the front traffic situation, as well as the multivehicle interactions effect in multiple vehicles following situation. So the LAS will lead to some complex phenomena. However, until now few scholars studied the effect of LAS on car following process.

In view of the reasons given above, we in this paper present a new car following model with considering the average speed of preceding cars. And a macro model for traffic flow is constructed by the relevancy relation of micro and macro variables. The analytical analysis and numerical simulation results indicate that the new consideration can stabilize the traffic flow.

\section{The Car Following Model}

The car following model focus on describing the motion of vehicles following each other on a single lane. In general, the motion equation is reduced as [3]

$$
\frac{d v_{j}(t)}{d t}=g\left(v_{j}, \Delta x_{j}, \Delta v_{j}\right),
$$

where $v_{j}$ and $x_{j}$ are, respectively, the velocity and position of the $j$ th car. $\Delta v_{j}=v_{j+1}-v_{j}$ denotes the relative velocity. $\Delta x_{j}=$ $x_{j+1}-x_{j}$ represents the space headway between two successive vehicles. The stimulus function $g(\cdot)$ depends on the factors 
velocity $v_{j}$, relative velocity $\Delta v_{j}$, and headway $\Delta x_{j}$. In this general framework, many classical car following models [46] have been developed and several important achievements have been attained.

Recently, in order to improve the stability of traffic system, scholars developed some extended car following models by incorporating multivehicle consideration in real traffic. Some were extended by introducing multiple information of headway $[7,8]$ or relative velocity $[9,10]$, whereas others considered both factors at the same time [11-13]. In 2005, considering the multiple headway information, Ge et al. [7] proposed the cooperative driving (CD) model with

$$
\frac{d v_{j}(t)}{d t}=g\left(v_{j}, \Delta x_{j}, \Delta x_{j+1}, \ldots, \Delta x_{j+n}\right) .
$$

In 2006, Wang et al. [9] developed a multiple velocity difference (MVD) model; that is,

$$
\frac{d v_{j}(t)}{d t}=g\left(v_{j}, \Delta x_{j}, \Delta v_{j}, \Delta v_{j+1}, \ldots, \Delta v_{j+k}\right) .
$$

In 2010, Peng and Sun [11] presented the multiple car following (MCF) model with simultaneously taking the multiple velocity difference and multiple headway information into account; that is,

$$
\begin{gathered}
\frac{d v_{j}(t)}{d t}=g\left(v_{j}, \Delta x_{j}, \Delta x_{j+1}, \ldots, \Delta x_{j+p}, \Delta v_{j},\right. \\
\left.\Delta v_{j+1}, \ldots, \Delta v_{j+p}\right) .
\end{gathered}
$$

Stability analysis in the aforementioned models all turn out that the stability of traffic flow is greatly improved by incorporating multivehicle consideration.

The aforementioned models can describe some complex traffic phenomena (e.g., congestion, instability, and stop-andgo waves in traffic flow). However, these models are unsuited to study the average speed effect of preceding vehicles group because they do not consider this factor at all. In fact, the average speed of preceding vehicles group reflects the whole front traffic situation on segment, that is, whether the traffic flow of preceding cars on segment will cluster, dissipate, or simply maintain a constant velocity. By applying T-CPS, the follower can sense the front traffic situation through the indicator of average speed of preceding vehicles group; then the following one can make a decision and adjust its vehicle in advance to adapt the state change of preceding cars based on sensing information. In view of the above reason, we developed a new car following model that considers the average speed information of preceding vehicles group. The model's dynamics equation is as follows:

$$
\frac{d v_{j}(t)}{d t}=a\left[V\left(\Delta x_{j}(t)\right)-v_{j}(t)\right]+\lambda\left[\bar{v}_{j}(t)-v_{j}(t)\right],
$$

where the term $\bar{v}_{j}(t)=(1 / n) \sum_{l=1}^{n} v_{j+l}(t)$ is the average speed of preceding vehicles group, which reflect the front traffic situation consisting of car $j$ and its leading cars $j, j+$ $1, \ldots, j+n$ at time $t . n$ denotes the number of the vehicles ahead considered. $\lambda$ is the responding factor to the difference between the velocity $v_{j}(t)$ and average speed term $\bar{v}_{j}(t)$. The idea of the extended model is that the acceleration of the $j$ th car is determined not only by the velocity $v_{j}(t)$ and the headway $\Delta x_{j}(t)$, but also by the velocity difference between velocity $v_{j}(t)$ and front local average speed $\bar{v}_{j}(t)$ at time $t$. When $n=1$, the new model is reduced to the FVD model [6].

\section{The Macro Model}

In order to construct the corresponding macro model, we should transform the micro variables of individual vehicles in (5) into the macro ones with using the method by Liu et al. [14]; that is,

$$
\begin{aligned}
& v_{j}(t) \longrightarrow v(x, t), v_{j+l}(t) \longrightarrow v(x+l \Delta, t), \\
& V\left(\Delta x_{j}\right) \longrightarrow v_{e}(\rho(x, t)), \\
& a \longrightarrow \frac{1}{T}, \quad \lambda \longrightarrow \frac{1}{\tau}
\end{aligned}
$$

where the $v(x, t), \rho(x, t)$ are the speed and density at the point $(x, t)$, respectively; $\Delta$ denotes the distance between the following and leading vehicles; $v_{e}(\rho)$ is the equilibrium speed; $T$ is the relaxation time and $\tau$ is the time needed for the backward propagated disturbance to travel a distance of $\Delta$. Thus, (5) can be rewritten as follows:

$$
\frac{\partial v}{\partial t}+v \frac{\partial v}{\partial x}=\frac{v_{e}-v}{T}+\frac{1}{\tau}\left[\sum_{l=1}^{n} \frac{v(x+l \Delta, t)}{n}-v(x, t)\right]
$$

Expanding (7), neglecting the nonlinear terms, and combining with the conservation equation of traffic flow, we can obtain a new macro model with considering the local average speed information of preceding vehicles group:

$$
\begin{gathered}
\frac{\partial \rho}{\partial t}+v \frac{\partial \rho}{\partial x}+\rho \frac{\partial v}{\partial x}=0 \\
\frac{\partial v}{\partial t}+\left[v-\frac{1}{2}(n+1) c_{0}\right] \frac{\partial v}{\partial x}=\frac{v_{e}-v}{T}
\end{gathered}
$$

where $c_{0}=\Delta / \tau$ denotes the propagating speed of the small perturbation. When $n=1$, the new model reduced to the speed gradient (SG) model [15]. Comparing our model with SG model, we can see that our model differs from the SG model in the motion equation, in which the number of preceding cars $n$ is involved.

In order to analyze the characteristic speed problem for the new model, we rewrite (8) in a vector forms as follows:

$$
\frac{\partial U}{\partial t}+A \frac{\partial U}{\partial x}=E
$$

where $U=\left[\begin{array}{l}\rho \\ v\end{array}\right], E=\left[\begin{array}{c}0 \\ \left(v_{e}-v\right) / T\end{array}\right]$, and $A=\left[\begin{array}{lc}v & \rho \\ 0 & v-(1 / 2)(n+1) c_{0}\end{array}\right]$.

The eigenvalues, $\lambda$, of the $A$ matrix are found by setting

$$
\operatorname{det}(A-\lambda I)=0 \text {, }
$$


where $I$ is identity matrix. From (10), one can obtain

$$
\lambda_{1}=v, \quad \lambda_{2}=v-\frac{1}{2}(n+1) c_{0} .
$$

Since $c_{0} \geq 0$, the characteristic speeds in (11) are no greater than the macroscopic flow velocity $v$. This result ensures that our model conforms to the anisotropy property which is the fundamental principle in single lane traffic flow $[15,16]$.

\section{Linear Stability Analysis}

Assuming that $\rho_{0}$ and $v_{0}=v_{e}\left(\rho_{0}\right)$ are the steady-state solutions for (8) and introducing the small perturbations $\xi=$ $\xi(x, t)$ and $\varepsilon=\varepsilon(x, t)$ into the steady-state solutions, one obtains the perturbed solution:

$$
\rho=\rho_{0}+\xi, \quad v=v_{0}+\varepsilon .
$$

Substituting the perturbed solutions (12) into (8) and then making the Taylor expansion at $\left(\rho_{0}, v_{0}\right)$ and neglecting higher-order terms of $\xi$ and $\varepsilon$, we obtain the following expression:

$$
\begin{gathered}
\xi_{t}+v_{0} \xi_{x}+\rho_{0} \varepsilon_{x}=0 \\
\varepsilon_{t}+\left[v_{0}-\frac{1}{2}(n+1) c_{0}\right] \varepsilon_{x}=\frac{v_{e}^{\prime}\left(\rho_{0}\right) \xi-\varepsilon}{T},
\end{gathered}
$$

where $\xi_{t}=d \xi / d t, \xi_{x}=d \xi / d x, \varepsilon_{t}=d \varepsilon / d t, \varepsilon_{x}=d \varepsilon / d x$, and $v_{e}^{\prime}=d v_{e} / d \rho$. Eliminating $\varepsilon$ from (13) results in the equation

$$
\left(\partial_{t}+c \partial_{x}\right) \xi=-T\left[\left(\partial_{t}+c_{1} \partial_{x}\right)\left(\partial_{t}+c_{2} \partial_{x}\right)\right] \xi,
$$

where $c=v_{e}^{\prime}\left(\rho_{0}\right) \rho_{0}+v_{0}, c_{1}=v_{0}-(1 / 2)(n+1) c_{0}$ and $c_{2}=v_{0}$. According to the traditional way of linear stability analysis, substituting $\xi(x, t)=\xi_{0} \exp i(\gamma x-w t)$ into (14), one can obtain

$$
(-i w+i c \gamma) \xi=-T\left[\left(-i w+c_{1} i \gamma\right)\left(-i w+c_{2} i \gamma\right)\right] \xi .
$$

For $\xi$ to be the nontrivial solution of (15), we must have

$$
T\left(w-c_{1} \gamma\right)\left(w-c_{2} \gamma\right)+i(w-c \gamma)=0 .
$$

It is obvious that the solution is stable if and only if the imaginary part of both of the roots $w$ is nonpositive. According to the method using in [15], the requirement for this is

$$
c_{1} \leq c \leq c_{2}
$$

When condition (17) cannot be met, unstable traffic will appear and lead to many complex traffic phenomena like stop-go waves or cluster jams.

\section{Numerical Simulation}

For numerical computations, we adopt the finite difference method to discretize (10), and the corresponding difference equations are as follows:

$$
\rho_{i}^{k+1}=\rho_{i}^{k}+\frac{\Delta t}{\Delta x} \rho_{i}^{k}\left(v_{i}^{k}-v_{i+1}^{k}\right)+\frac{\Delta t}{\Delta x} v_{i}^{k}\left(\rho_{i-1}^{k}-\rho_{i}^{k}\right) .
$$

If the traffic is heavy, $v_{i}^{k}<(1 / 2)(n+1) c_{0}$,

$$
v_{i}^{k+1}=v_{i}^{k}+\frac{\Delta t}{\Delta x}\left[\frac{(n+1) c_{0}}{2}-v_{i}^{k}\right]\left(v_{i+1}^{k}-v_{i}^{k}\right)+\frac{\Delta t}{T}\left(v_{e}-v_{i}^{k}\right) .
$$

Otherwise

$$
v_{i}^{k+1}=v_{i}^{k}+\frac{\Delta t}{\Delta x}\left[\frac{(n+1) c_{0}}{2}-v_{i}^{k}\right]\left(v_{i}^{k}-v_{i-1}^{k}\right)+\frac{\Delta t}{T}\left(v_{e}-v_{i}^{k}\right),
$$

where $i, k, \Delta x$, and $\Delta t$ are the space index, the time index, the spatial step, and the time step, respectively.

First, we use numerical tests to investigate whether our model can capture the shock and rarefaction wave of the real traffic flow. The initial conditions are as follows $[15,17]$ :

$$
\begin{array}{ll}
\rho_{u}^{1}=0.04(\mathrm{veh} / \mathrm{m}), \quad \rho_{d}^{1}=0.18(\mathrm{veh} / \mathrm{m}), \\
\rho_{u}^{2}=0.18(\mathrm{veh} / \mathrm{m}), \quad \rho_{d}^{2}=0.04(\mathrm{veh} / \mathrm{m}),
\end{array}
$$

where $\rho_{u}^{1}$ and $\rho_{d}^{1}$ are the upstream and downstream densities, respectively, in the case of shock wave; $\rho_{u}^{2}$ and $\rho_{d}^{2}$ are the upstream and downstream densities, respectively, in the case of rarefaction wave. The initial speeds are set below:

$$
v_{u}^{1,2}=v_{e}\left(\rho_{u}^{1,2}\right), \quad v_{d}^{1,2}=v_{e}\left(\rho_{d}^{1,2}\right) .
$$

Comparing with the results of [15], we adopt the following equilibrium speed here [18]:

$$
v_{e}(\rho)=v_{f}\left[1-\exp \left(1-\exp \left(\frac{c_{m}}{v_{f}}\left(\frac{\rho_{j}}{\rho}-1\right)\right)\right)\right],
$$

where $v_{f}$ is the free speed and $c_{m}$ the kinematic wave speed at jam density $\rho_{j}$. Here, the free boundary conditions are adopted, that is, $\partial v / \partial x=0$ and $\partial \rho / \partial x=0$ on both sides. And $20 \mathrm{~km}$ test road section is divided into 100 meshes equally. Other inputting parameters are as follows: $v_{f}=30 \mathrm{~m} / \mathrm{s}$, $c_{m}=c_{0}=11 \mathrm{~m} / \mathrm{s}, T=10 \mathrm{~s}, \rho_{j}=0.2 \mathrm{veh} / \mathrm{m}, \Delta x=200 \mathrm{~m}$, and $\Delta t=1 \mathrm{~s}$.

The computational results for the new model $(n=3)$ under the initial conditions of (20a) and (20b) are shown in Figures 1(a) and 1(b), respectively. Equation (20a) simulates the free-flow traffic meets a queue of nearly stopping vehicles where shock waves will appear while (20b) describes the rarefaction wave corresponding to that in the process of dissolution. Figures 1(a) and 1(b) show the density evolution in shock and rarefaction waves, respectively; the results are similar to those reported in $[15,17]$, so our model can provide correct predictions in the formation and propagation of the waves. In addition, owing to the local average speed of preceding cars considered in our model, the shock front between the congested and the free-flow traffic is smoother than that in [15], which means that the new consideration affects the smoothness of shock front positively.

In order to further study the effect of average speed of preceding cars on traffic flow, next, we study the evolution 


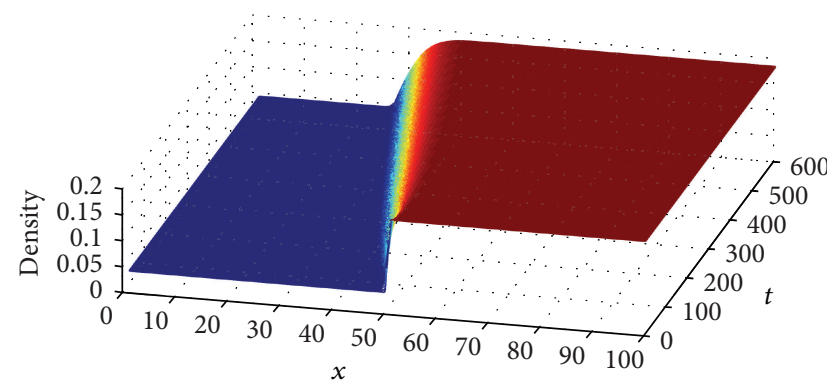

(a)

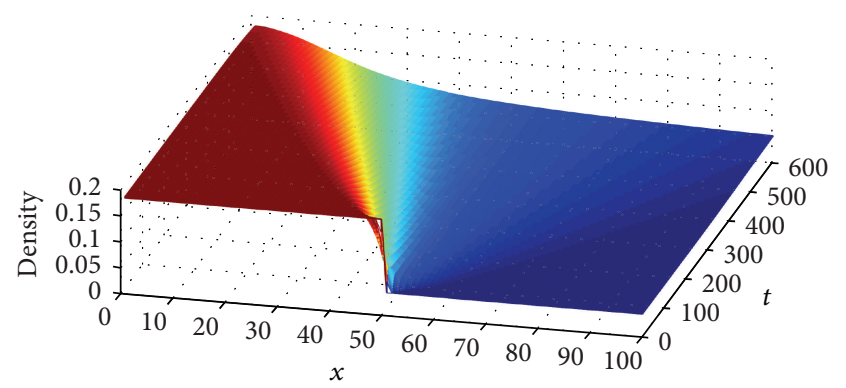

(b)

Figure 1: The density evolution for the new model $(n=3)$ under the initial conditions (22), where (a) shock wave corresponds to (20a) and (b) rarefaction wave corresponds to (20b).

of small disturbance with our model. In this paper, the initial condition is chosen as follows [19]:

$$
\begin{aligned}
\rho(x, 0)=\rho_{0}+\Delta \rho\left\{\cosh ^{-2}\left[\frac{160}{L}\left(x-\frac{5 L}{16}\right)\right]\right. \\
\left.-\frac{1}{4} \cosh ^{-2}\left[\frac{40}{L}\left(x-\frac{11 L}{32}\right)\right]\right\},
\end{aligned}
$$

where $L=32.2 \mathrm{~km}$ is the road length. Here the periodic boundary condition is adopted; that is,

$$
\rho(L, t)=\rho(0, t), \quad v(L, t)=v(0, t) .
$$

In order to compare with the results [15], we here use the following equilibrium speed-density relationship [20]:

$$
\begin{aligned}
v_{e}(\rho)=v_{f}\{ & \left(1+\exp \left[\frac{\rho / \rho_{\mathrm{jam}}-0.25}{0.06}\right]\right)^{-1} \\
& \left.-3.72 \times 10^{-6}\right\} .
\end{aligned}
$$

Assume that the initial flow is in local equilibrium everywhere; that is, $v(x, 0)=v_{e}(\rho(x, 0))$. Let $\Delta \rho=0.01 \mathrm{veh} / \mathrm{m}$, $\Delta x=100 \mathrm{~m}$, and $\Delta t=1 \mathrm{~s}$ in the following simulations, and the other parameters are the same as in Figure 1.

When $n=3$, the unstable region $0.04<\rho_{0}<0.07$ for our model can be obtained by substituting the parameters into (17). In comparison with the unstable region $0.031<$ $\rho_{0}<0.084$ that is reported in SG model, one can find out that the unstable area is shrunk in our model compresses by considering the average speed information of preceding cars. This means that our model has effectively improved the stability of traffic flow. Under different initial densities $\rho_{0}$, the corresponding numerical results for our model with $n=3$ are shown in Figure 2.

In Figure 2(a), because density is very low, the perturbation in the traffic flow dissipates quickly with time and the traffic flow is stable as we expect. As the initial density increases and just exceeds the down-critical density, the small perturbations in Figure 2(b) were amplified gradually and backward propagating traffic jam occurs. When the density becomes higher and increases to 0.058 , in Figure 2(c), the traffic flow get worse and multiple clusters were formed, which correspond to the stop-and-go traffic phenomenon in the real world. Finally, when the density becomes greater than the up-critical density, it is obvious from Figure 2(d) that the traffic flow reaches stable state again. Therefore, the results of the simulation are in good agreement with those of the linear stability analysis.

Figure 3 shows density evolution of small perturbation with initial density $\rho_{0}=0.07$ for different values of $n$. Figures $3(\mathrm{a})$ and $3(\mathrm{~b})$ are corresponding to the cases of $n=1$ and $n=2$, respectively. From Figures 3 and 2(d), one can find out that as the value of $n$ increases, the amplitude of density wave weakens gradually, which further demonstrates that the average speed information of preceding vehicles group can effectively stabilize the traffic flow. Thus the new consideration plays an important role in traffic nonlinear dynamics features.

\section{Conclusions}

In recent years, wireless communication technology has been widely applied in T-CPS environment, and thus much more information is available for drivers than ever before. With the help of T-CPS, drivers can obtain the road average speed of preceding cars. However, few existing car following models directly study this factor. In this paper, we presented a new car following model that considers the effect of average speed of preceding vehicles group to stabilize the traffic flow. Starting from this car following model, we then develop a new macro traffic flow model via the relationship between the micro and macro variables. By means of linear stability theory, the stable criterion for macro model is obtained. The numerical tests and analytical results indicate that the presented macro model can not only describe the complex phenomena including rarefaction wave, shock wave, stopand-go traffic, and small perturbation effect, but also show that the new consideration is able to improve the stability of traffic flow greatly.

Furthermore, it should be noticed that, differentiating from the original FVD model which just considered the information of the nearest preceding car, the presented car following model focused on the effect that the interaction of multiple preceding cars has on the following behavior of 


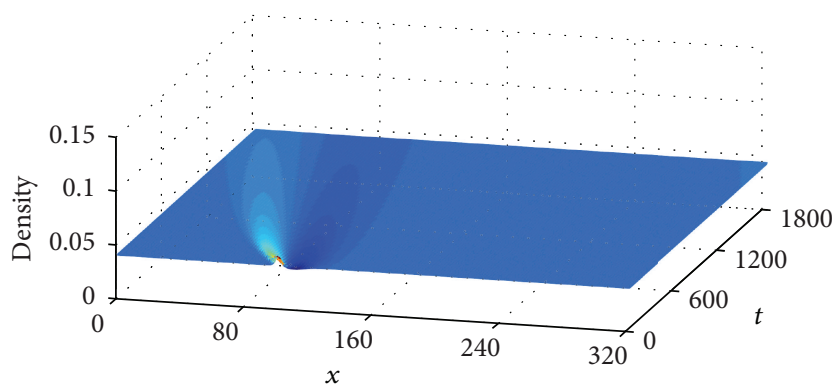

(a)

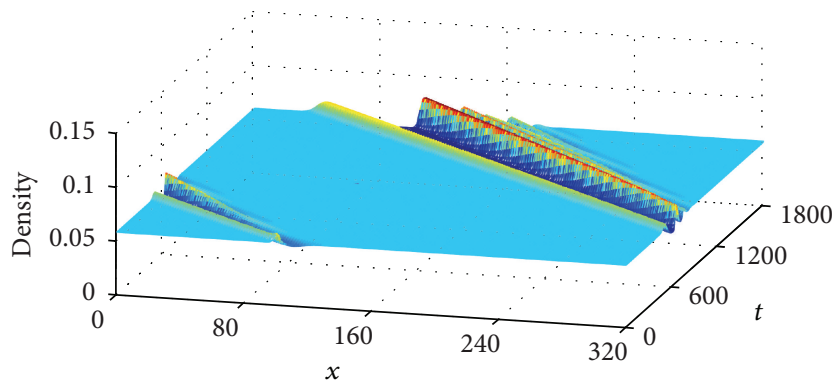

(c)

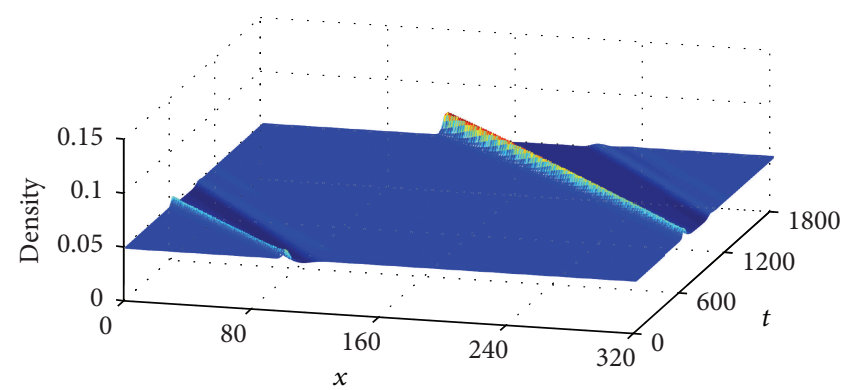

(b)

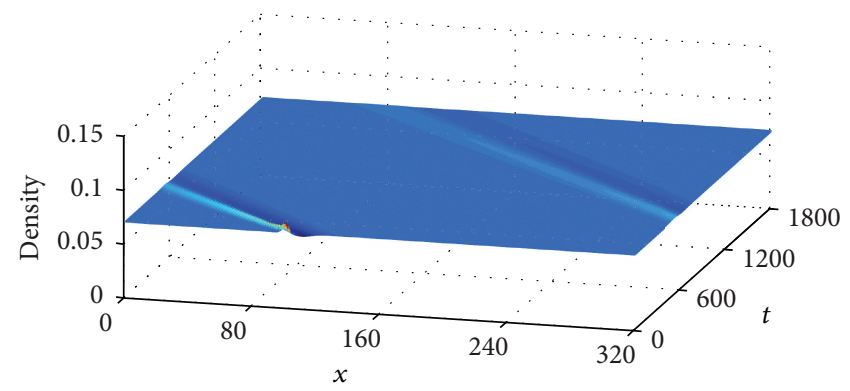

(d)

Figure 2: As $n=3$, the temporal evolution of traffic density for (a) $\rho_{0}=0.042$, (b) $\rho_{0}=0.049$, (c) $\rho_{0}=0.058$, and (d) $\rho_{0}=0.07$, where the units of the $t$-axis, the $x$-axis, and the density-axis are second, $100 \mathrm{~m}$, and veh/m, respectively.

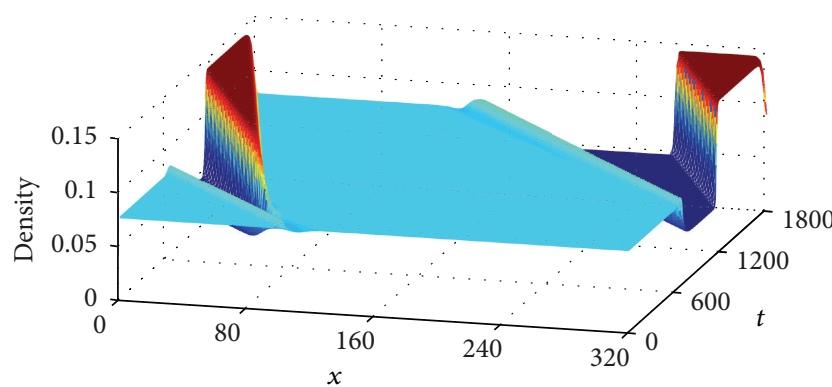

(a)

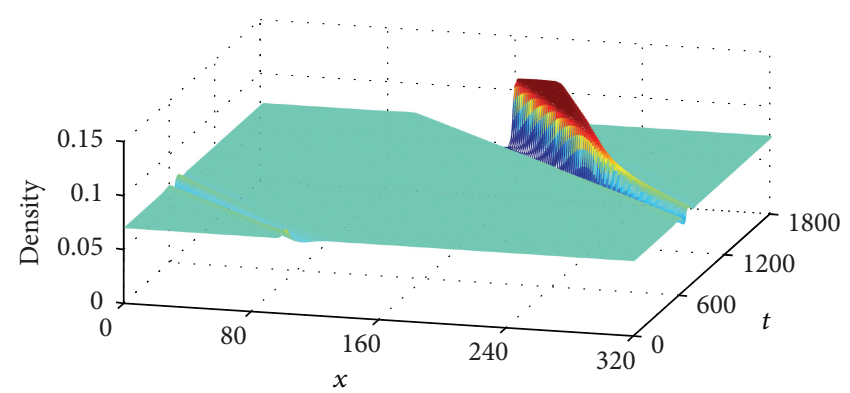

(b)

Figure 3: Temporal evolution of traffic density under initial density $\rho_{0}=0.07$ for (a) $n=1$ and (b) $n=2$, where the units of the $t$-axis, the $x$-axis, and the density-axis are second, $100 \mathrm{~m}$, and veh/m, respectively.

the host car. The average speed of preceding vehicles group is considered in the dynamic equation of the new car following model. When the number of vehicles in the group is just one, the presented car following model reduced into FVD model.

\section{Conflict of Interests}

The authors declare that there is no conflict of interests regarding the publication of this paper.

\section{Acknowledgments}

This work is supported by the Fundamental Research Funds for the Central Universities (Grant no. 106112014CDJZR178801), the Research Fund for the Doctoral Program of Higher Education of China (Grant no. 20120191110047), the Natural Science Foundation of Chongqing Science \& Technology Commission of China (Grant no. 2012jjB40002), the Planned Science and Technology Program of Chongqing, China (Grant no. cstc2012ggyyjs00006), and the Natural Science Foundation of Guangxi Zhuang Autonomous Region, China (Grant no. 2013GXNSFAA019347).

\section{References}

[1] B.-L. Lin, J.-W. Li, L.-J. Ji, and Y.-C. Huang, "A statistical theory to aggregation in one-dimensional freeway traffic," Journal of Statistical Physics, vol. 141, no. 6, pp. 1104-1115, 2010.

[2] M. Blank, "Ergodic properties of a simple deterministic traffic flow model," Journal of Statistical Physics, vol. 111, no. 3-4, pp. 903-930, 2003. 
[3] D. Chowdhury, L. Santen, and A. Schadschneider, "Statistical physics of vehicular traffic and some related systems," Physics Reports, vol. 329, no. 4-6, pp. 199-329, 2000.

[4] M. Bando, K. Hasebe, A. Nakayama, A. Shibata, and Y. Sugiyama, "Dynamical model of traffic congestion and numerical simulation,” Physical Review E, vol. 51, no. 2, pp. 1035-1042, 1995.

[5] D. Helbing and B. Tilch, "Generalized force model of traffic dynamics," Physical Review E, vol. 58, no. 1, pp. 133-138, 1998.

[6] R. Jiang, Q. S. Wu, and Z. J. Zhu, "Full velocity difference model for a car-following theory," Physical Review E, vol. 64, no. 1, Article ID 017101, 2001.

[7] H. X. Ge, S. Q. Dai, Y. Xue, and L. Y. Dong, "Stabilization analysis and modified Korteweg-de Vries equation in a cooperative driving system," Physical Review E, vol. 71, no. 6, Article ID 066119, 7 pages, 2005.

[8] K. Hasebe, A. Nakayama, and Y. Sugiyama, "Dynamical model of a cooperative driving system for freeway traffic," Physical Review E, vol. 68, no. 2, Article ID 026102, 2003.

[9] T. Wang, Z.-Y. Gao, and X.-M. Zhao, "Multiple velocity difference model and its stability analysis," Acta Physica Sinica, vol. 55, no. 2, pp. 634-640, 2006.

[10] H. X. Ge, R. J. Cheng, and Z. P. Li, "Two velocity difference model for a car following theory," Physica A: Statistical Mechanics and Its Applications, vol. 387, no. 21, pp. 5239-5245, 2008.

[11] G. H. Peng and D. H. Sun, "A dynamical model of car-following with the consideration of the multiple information of preceding cars," Physics Letters, Section A: General, Atomic and Solid State Physics, vol. 374, no. 15-16, pp. 1694-1698, 2010.

[12] Y. Li, D. Sun, W. Liu et al., "Modeling and simulation for microscopic traffic flow based on multiple headway, velocity and acceleration difference," Nonlinear Dynamics, vol. 66, no. 1-2, pp. 15-28, 2011.

[13] Y. M. Hu, T. S. Ma, and J. Z. Chen, "An extended multianticipative delay model of traffic flow," Communications in Nonlinear Science and Numerical Simulation, vol. 19, no. 9, pp. 3128-3135, 2014.

[14] G. Q. Liu, A. S. Lyrintiz, and P. G. Michalopoulos, "Improved high-order model for freeway traffic flow," Transportation Research Record, vol. 1644, pp. 37-46, 1998.

[15] R. Jiang, Q.-S. Wu, and Z.-J. Zhu, "A new continuum model for traffic flow and numerical tests," Transportation Research Part B: Methodological, vol. 36, no. 5, pp. 405-419, 2002.

[16] C. F. Daganzo, "Requiem for second-order fluid approximations of traffic flow," Transportation Research Part B, vol. 29, no. 4, pp. 277-286, 1995.

[17] T. Q. Tang, H. J. Huang, and H. Y. Shang, "A new macro model for traffic flow with the consideration of the driver's forecast effect," Physics Letters A, vol. 374, no. 15-16, pp. 1668-1672, 2010.

[18] J. M. Del Castillo, P. Pintado, and F. G. Benitez, "The reaction time of drivers and the stability of traffic flow," Transportation Research Part B, vol. 28, no. 1, pp. 35-60, 1994.

[19] M. Herrmann and B. S. Kerner, "Local cluster effect in different traffic flow models," Physica A: Statistical Mechanics and Its Applications, vol. 255, no. 1-2, pp. 163-188, 1998.

[20] B. S. Kerner and P. Konhäuser, "Cluster effect in initially homogeneous traffic flow," Physical Review E, vol. 48, no. 4, pp. R2335-R2338, 1993. 


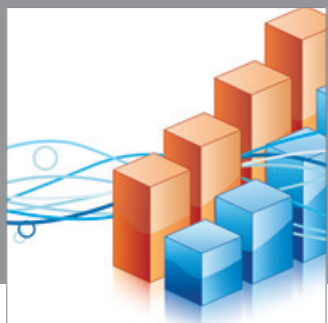

Advances in

Operations Research

mansans

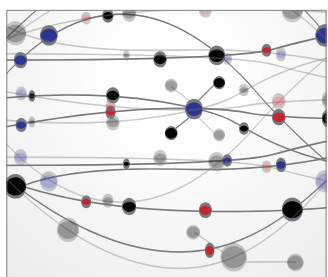

The Scientific World Journal
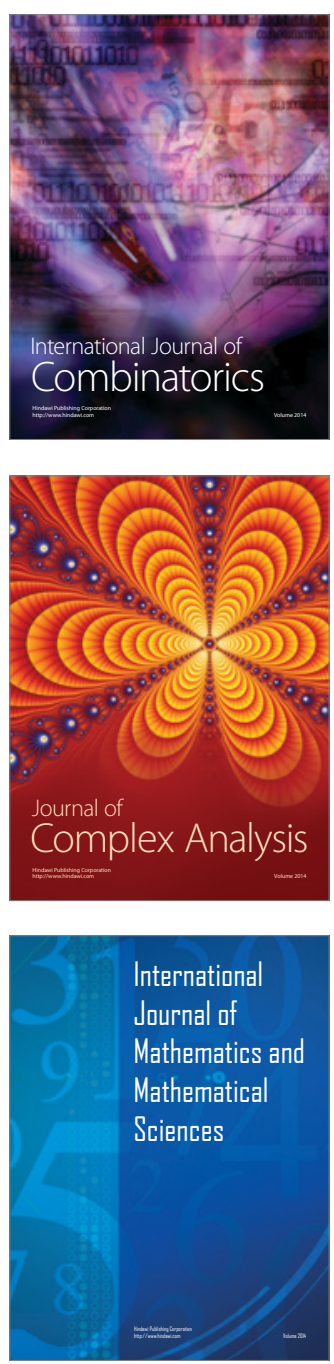
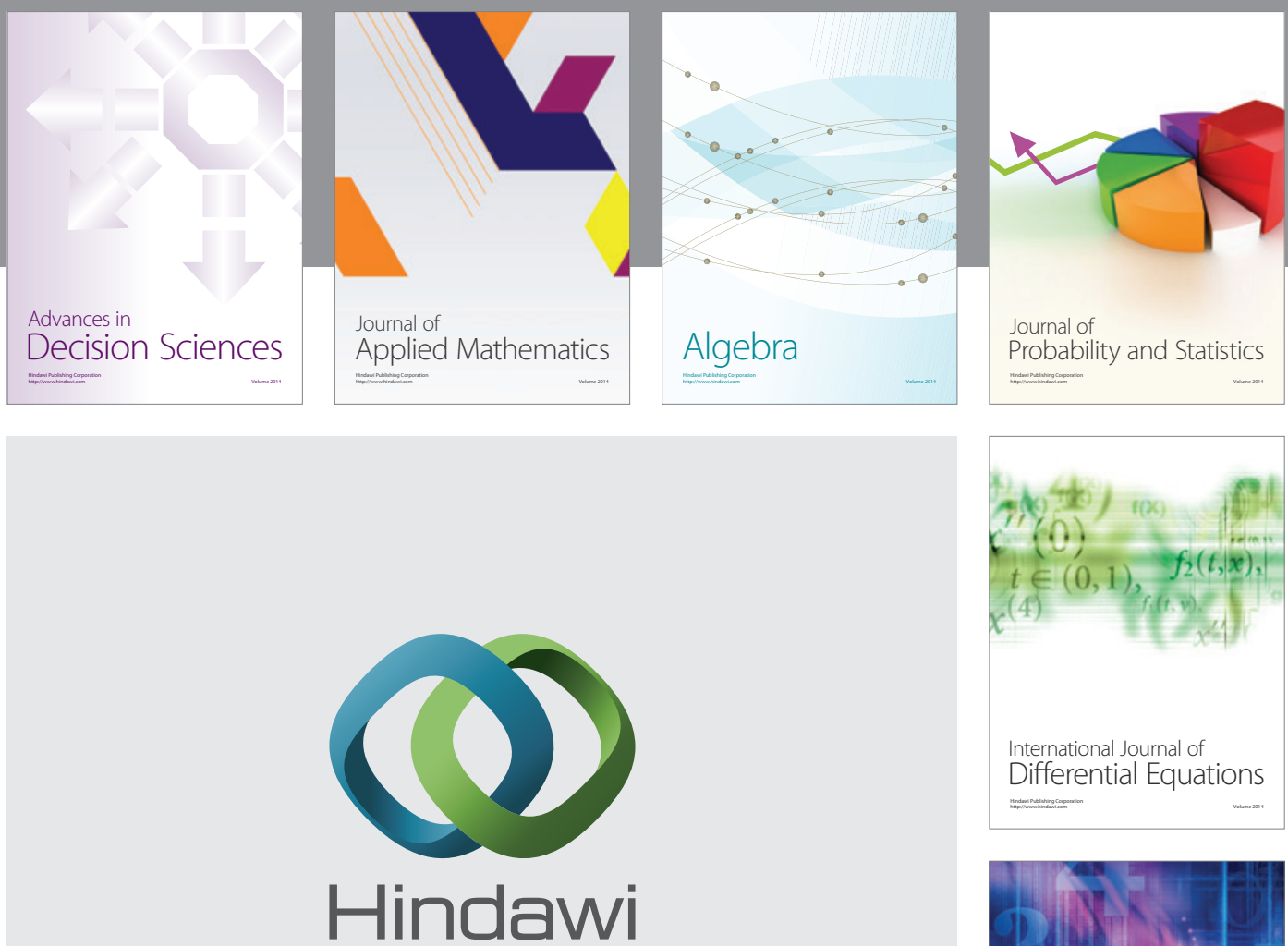

Submit your manuscripts at http://www.hindawi.com
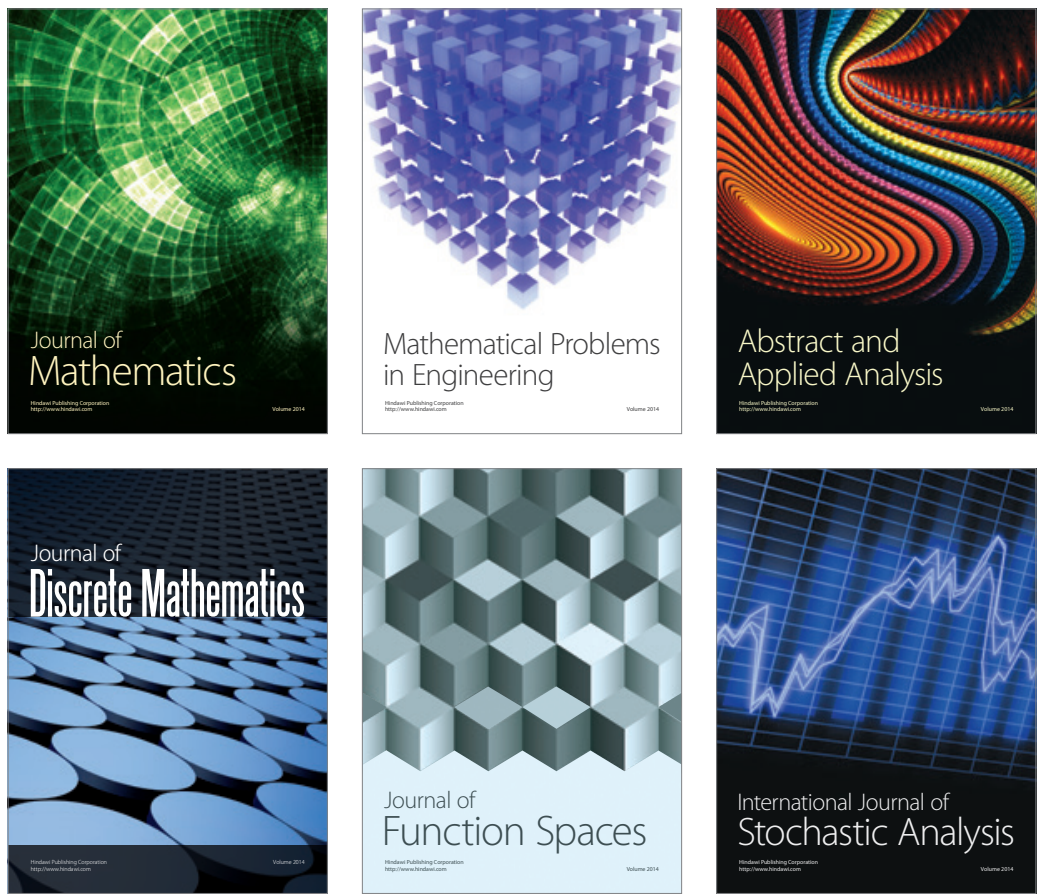

Journal of

Function Spaces

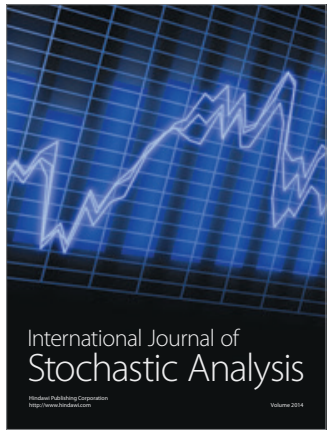

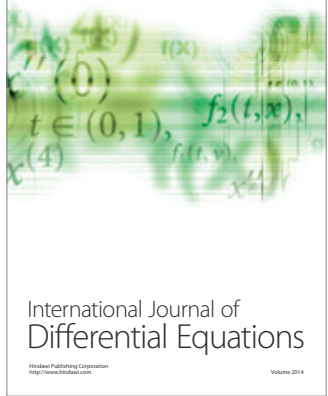
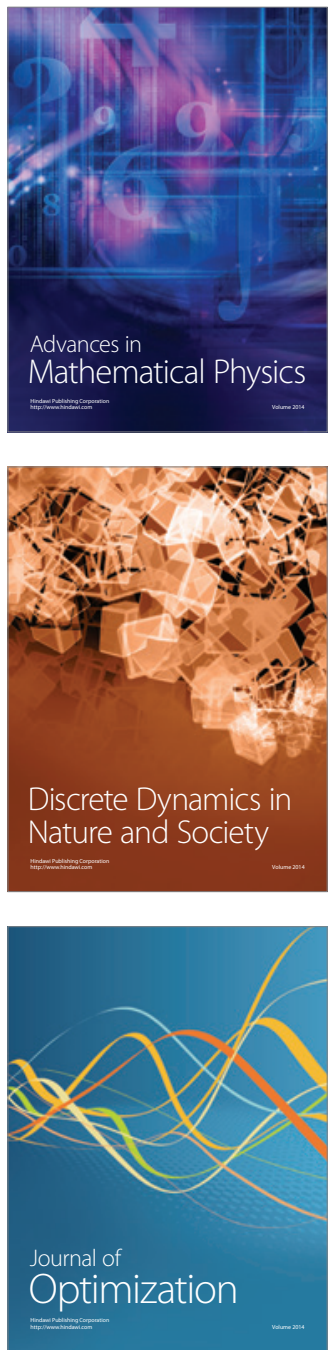